\title{
Future of Decellularized Dental Pulp Matrix in Regenerative Endodontics
}

\author{
Zohaib Khurshid ${ }^{10}$ Ahmed Jamil Ahmed Alnaim² \\ Eisha Imran ${ }^{3}$ Necdet Adanir ${ }^{4}$
}

Ahmed Abdulhakim Ahmed Alhashim²

Address for correspondence Zohaib Khurshid, BDS, MRes, FHEA, MFDS RCPS, MDTFEd, Department of Prosthodontics and Dental Implantology, College of Dentistry, King Faisal University, Al-Ahsa 31982, Kingdom of Saudi Arabia (e-mail: zsultan@kfu.edu.sa).

\author{
Abstract \\ Keywords \\ - tooth \\ - dental pulp tissue \\ - root canal treatment \\ - decellularization \\ - regenerative \\ endodontics
}

With the advancements in tissue engineering, the repair and regeneration of oral/dental tissue are becoming possible and productive. Due to periodontal diseases, the tooth loses bone support resulting in tooth loss, but bone grafting stabilizes with new bone. It is seen that due to the progression of dental caries, pulp damage happens, and the vitality of the tooth is compromised. The current theme of dental pulp regeneration through biological and synthetic scaffolds, is becoming a potential therapy for pulp revitalization.

\section{Introduction}

Dental pulp can be considered as the heart of a tooth. Its composition is complex and consists of organic and inorganic material, including connective tissue, blood vessels, and a cluster of different types of cells. ${ }^{1}$ Dental pulp has a significant role in the integrity of the tooth, tooth vitality, providing the tooth with nutrients through blood vessels, and aiding the formation of dentin in the case of tissue damage through the formation of odontoblast cells. ${ }^{2}$ The pulp is richly innervated with different types of nerves, including sensory, sympathetic, and parasympathetic nerve fibers. The sensory fibers have a significant role as they act as an alarm system if there is an inflammation or any pulp damage is present. Pain is mediated mainly with two different sensory fibers: A-delta fibers, which are myelinated and moderately fast in conduction that convey sharp pain and cold sensation, and C-fibers, which are nonmyelinated, slow in conduction, and convey dull pain. ${ }^{3}$

published online January 6, 2022
DOI https://doi.org/ 10.1055/s-0041-1741012. ISSN 1305-7456.
Dental caries represent one of the principal challenges to the health of the dental pulp, although its treatment may well exacerbate the challenge. ${ }^{4} \mathrm{~A}$ fundamental consideration in pulp protection is recognizing that infection is a crucial driver of inflammation, which often determines the outcomes for pulp survival. Thus, the pulp always is likely to be inflamed when bacteria from dental caries are present, and their control should be a significant feature of any treatment planning. Regarding the conventional intervention to treat damaged pulp tissue, some drawbacks might affect the prognosis of the tooth even if the root canal treatment is done ideally. The tooth is mainly affected structurally as the tooth is eliminated of living tissues, debrided of necrotic dentin, and the canal spaces become widened, thus leaving the tooth dehydrated and fragile. It is important for the pulp to be healthy to perform its functions such as nutrient supply to the teeth, dentine formation, sensing, defense, and other physiological functions. The future of endodontics aims to

\section{(c) 2022. The Author(s).}

This is an open access article published by Thieme under the terms of the Creative Commons Attribution License, permitting unrestricted use, distribution, and reproduction so long as the original work is properly cited. (https://creativecommons.org/licenses/by/4.0/)

Thieme Medical and Scientific Publishers Pvt. Ltd., A-12, 2nd Floor, Sector 2, Noida-201301 UP, India 
ensure pulp vitalization by pulp tissue regeneration, using xenograft-derived stem cells to accomplish cell proliferation that could replace the diseased pulp tissue with healthy ones, regaining the structural and biological integrity of the pulp and tooth structure. ${ }^{3,5,6}$

Maintenance of pulp vitality should always be the goal in treatment planning, and considerable interest is developing in the concept of regenerative endodontics for complete or partial pulp tissue regeneration. ${ }^{7}$ A new pathway has been opened due to advancement in regenerative medicine by transplanting stem cells along with the growth factors and a biological scaffold into the prepared pulp cavity. These stem cells can proliferate and differentiate into various cells in the pulp to achieve functional pulp regeneration. ${ }^{1,3,5}$ Basically, regenerative endodontics has been pioneered by the experimental studies of Nygaard-Ostby and Hjortdal, involving induced bleeding from the periapical tissues into the chemo-mechanically debrided canal space of teeth, which was partly filled with root filling. ${ }^{8}$

Regeneration is a process by which altered tissues are entirely replaced by tissues native to their original architecture and function. ${ }^{9}$ It is based on the concept of tissue engineering technology that regenerates the dentine pulp complex in the canal space of immature permanent teeth, which could either be damaged by caries or trauma, and restores development of the arrested tooth root.

\section{Decellularization of Dental Pulp Tissue}

Decellularization can be described as a procedure that aims to remove cellular contents of a tissue, leaving the extracellular matrix (ECM) free of antigens that could produce an inflammatory reaction, thus reducing the risk of spread of disease as well as leaving the tissue in its original threedimensional biostructure. ${ }^{10}$ The process of decellularization is complex and can be achieved in different ways; it can be done mechanically, chemically/enzymatically, or by combining these methods. This is done to preserve the nanostructure environment of fibrous and adhesive proteins, which will aid in cell anchorage and regulate cellular activities in the future. By doing so, we can achieve local resident cell support, which can be a big step in pulp/dentine tissue engineering. In - Fig. 1, diagrammatic representation of the decellularization of dental pulp tissues for regenerative endodontics has been provided. There are many procedures and protocols that are reported for the decellularization of the dental pulp tissues, as mentioned in -Table 1.

It is also seen that regeneration of a pulp structure is usually unlikely to be successful. Still, if basic principles of tissue engineering are implemented within any regenerative endodontic procedures, it may seem to work. Also, reviews of regenerative endodontic procedures, known as revitalization procedures, have concluded that the outcomes are very unpredictable. ${ }^{16}$ In - Table 1, description of the decellularization process reported by the researchers for the extraction of bioactive ingredients from dental pulp tissues of different sources has been given. ${ }^{11-15}$

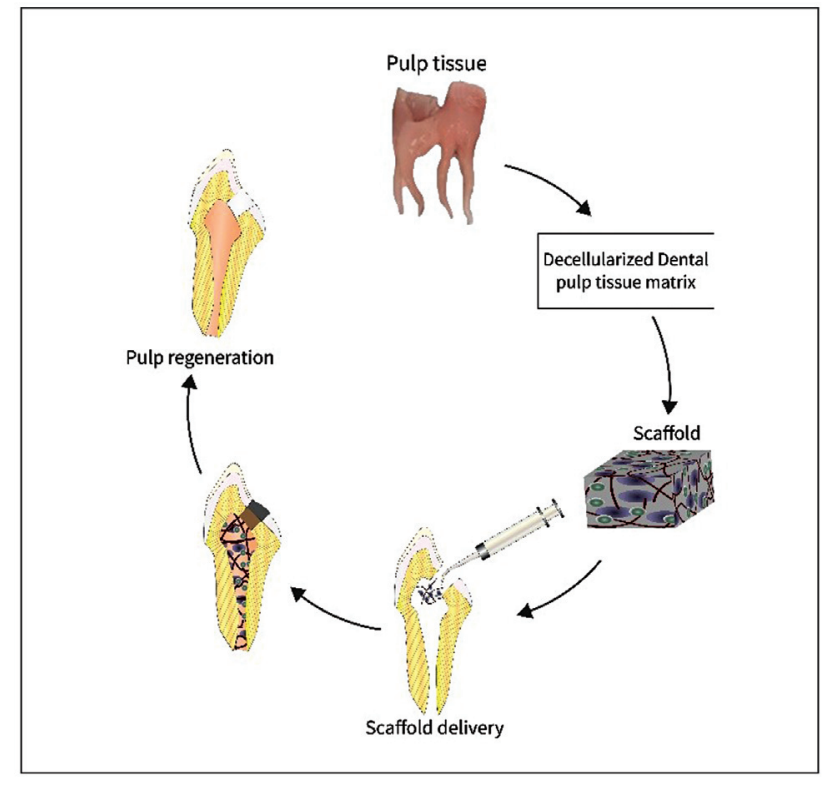

Fig. 1 Diagrammatic representation of the preparation of dental pulp tissues for regenerative dentistry.

Recent discoveries in endodontic dentistry have proven that the human dental pulp is capable of regeneration by using the methods of decellularization by making use of the dental pulp as a scaffold for the potential regeneration process. ${ }^{9}$ The human dental pulp is known to contain a variety of cells, primarily identified as stem cells that have a potential to induce differentiation to multiple classes of different cells, including osteoblasts, odontoblasts, neural cells, and adipocytes. Decellularized extracellular matrix ( $\mathrm{dECM}$ ) derived from either human or animal tissues has been considered as a possible scaffolding medium for tissue regeneration in present studies. ${ }^{11} \mathrm{~A}$ study has proven that $\mathrm{dECM}$ extracted from dental pulps can stimulate differentiation in periodontal ligament stem cells and in bone marrow, stromal cells, without any other external contributing factors. Another study, done by Song et al, demonstrated that the process of proliferation of dental pulp stem cells from the apical papilla and their differentiation to odontoblastic cells was assisted by the decellularized human dental pulp. ${ }^{14}$

On the other hand, they also found that the scaffold was permanently fixed in shape. It was difficult to insert into narrow and oblique canals of the roots, restricting their potential advantage in clinical application, thus resulting in limited success. Until now, there are no well-founded scaffolds that can mimic the complex ECM of the dental pulp. Therefore, it results in a challenge in producing a regenerative microenvironment for stem cell differentiation. - Fig. 2 discusses the benefits of using decellularized dental pulp tissue matrix as a bioscaffold.

\section{Conclusion}

The dental pulp is a unique tissue that contains specialized resident cells, stem cells, and immune cells that contribute to the pulp's defense strategy. It is seen that dental pulp has a 
Dental Pulp Matrix and Regenerative Endodontics Khurshid et al. 739

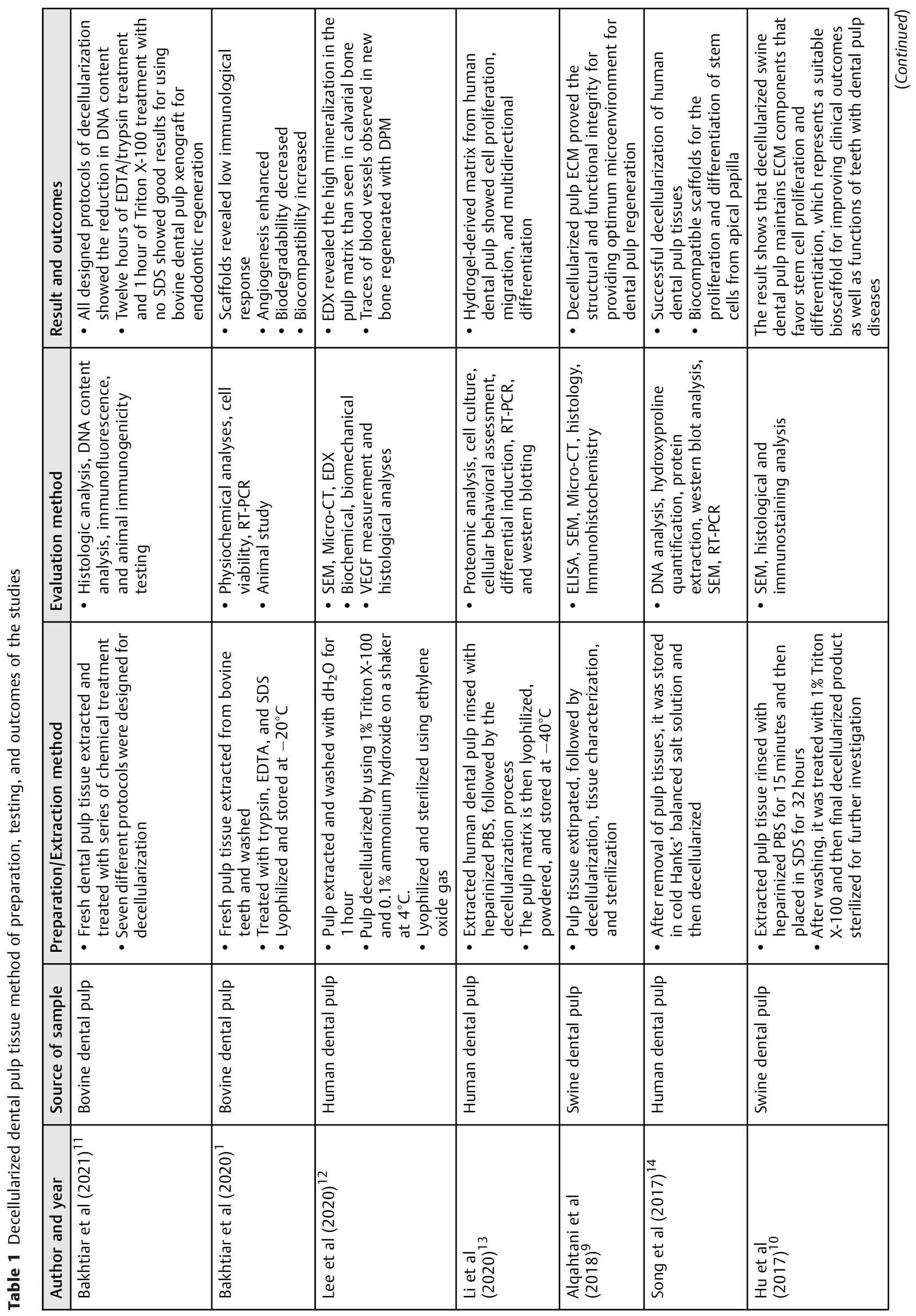




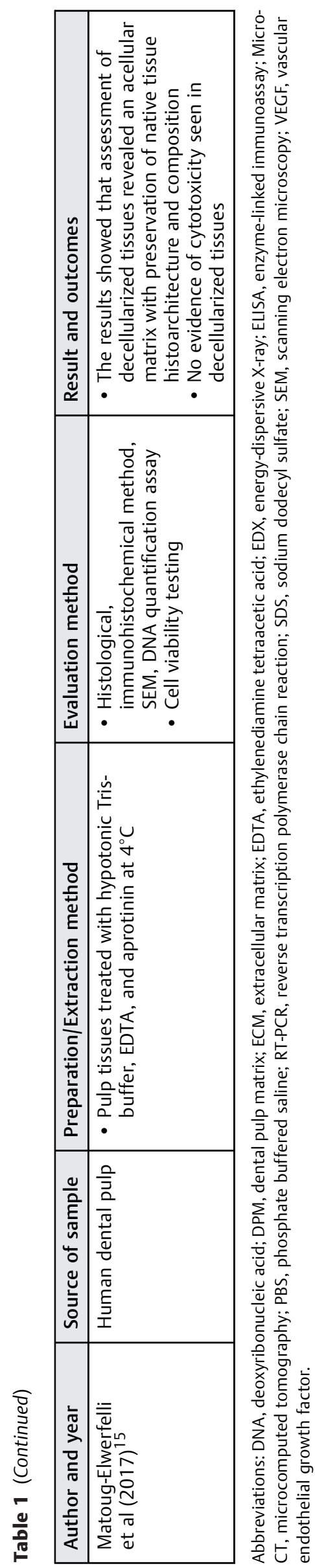

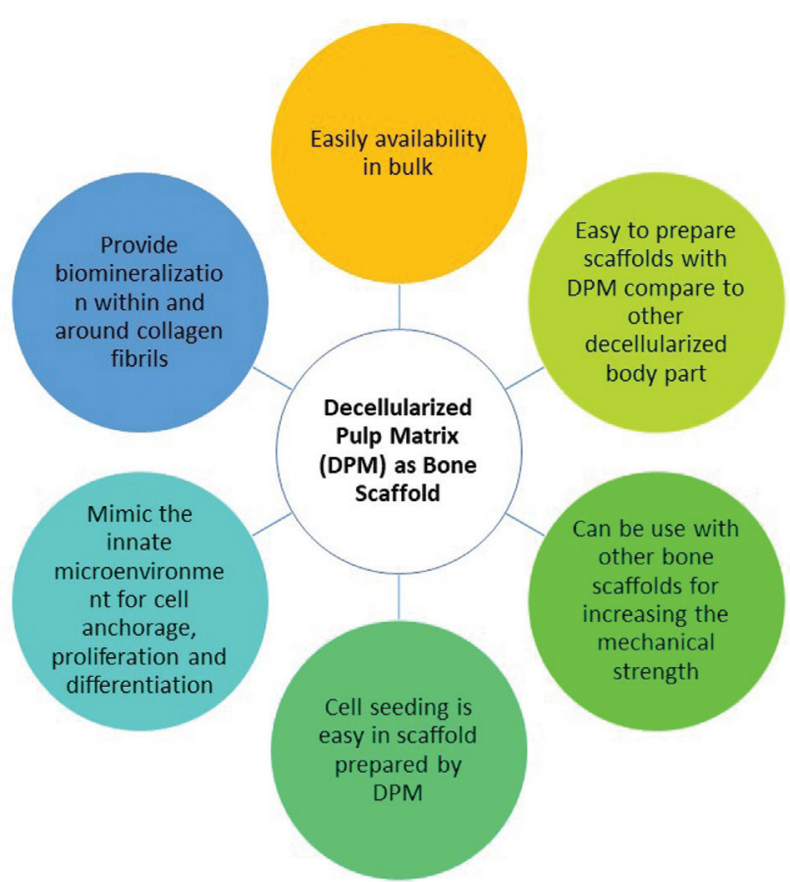

Fig. 2 Advantages of dental pulp matrix as a scaffold for bone tissue engineering.

substantial ECM that helps maintain the tissue's integrity. Due to their broad activity against a range of pathogens, it is known that novel peptide-based therapeutics are more effective against polymicrobial infections such as endodontic infections. It is believed that it is possible to develop a decellularized biocompatible biological scaffold containing the native ECM structural components that are required for tissue-specific regeneration. It is also seen that regenerative endodontics opens exciting opportunities for the preservation of pulp vitality, which underwent episodes of trauma and disease, and can encourage continued root maturation of immature permanent teeth with necrotic pulp. Overall, it is seen that the possibility to decellularize healthy dental pulp does open new horizons in regenerative dentistry as these decellularized tissues could help in serving as natural scaffolds.

\section{Funding}

None.

Conflict of Interest

None declared.

\section{References}

1 Bakhtiar H, Pezeshki-Modaress M, Kiaipour Z, et al. Pulp ECMderived macroporous scaffolds for stimulation of dental-pulp regeneration process. Dent Mater 2020;36(01):76-87

2 Zhang X, Li H, Sun J, et al. Cell-derived micro-environment helps dental pulp stem cells promote dental pulp regeneration. Cell Prolif 2017;50(05):e12361. Doi: 10.1111/cpr.12361

3 Smith AJ. Reflections and future visions for pulp biology research. J Endod 2020;46(9S):S42-S45 
4 Paduano F, Marrelli M, White LJ, Shakesheff KM, Tatullo M. Odontogenic differentiation of human dental pulp stem cells on hydrogel scaffolds derived from decellularized bone extracellular matrix and collagen type I. PLoS One 2016;11(02):e0148225. Doi: 10.1371/journal.pone.0148225

5 Simon S, Smith AJ. Regenerative endodontics. Br Dent J 2014;216 (06):E13. Doi: 10.1038/sj.bdj.2014.243

6 Zafar MS, Khurshid Z, Almas K. Oral tissue engineering progress and challenges. Tissue Eng Regen Med 2015;12(06):387-397

7 Kim SG, Malek M, Sigurdsson A, Lin LM, Kahler B. Regenerative endodontics: a comprehensive review. Int Endod J 2018;51(12): 1367-1388

8 Nygaard-Ostby B, Hjortdal O. Tissue formation in the root canal following pulp removal. Scand J Dent Res 1971;79(05):333-349

9 Alqahtani Q, Zaky SH, Patil A, Beniash E, Ray H, Sfeir C. Decellularized swine dental pulp tissue for regenerative root canal therapy. J Dent Res 2018;97(13):1460-1467

$10 \mathrm{Hu} \mathrm{L}, \mathrm{Gao} \mathrm{Z}, \mathrm{Xu} \mathrm{J}$, et al. Decellularized swine dental pulp as a bioscaffold for pulp regeneration. BioMed Res Int 2017; 2017:9342714. Doi: 10.1155/2017/9342714
11 Bakhtiar H, Rajabi S, Pezeshki-Modaress M, et al. Optimizing methods for bovine dental pulp decellularization. J Endod 2021;47(01):62-68

12 Lee DJ, Miguez P, Kwon J, et al. Decellularized pulp matrix as scaffold for mesenchymal stem cell mediated bone regeneration.J Tissue Eng 2020;11:2041731420981672. Doi: 10.1177/2041731 4209816722

13 Li J, Rao Z, Zhao Y, et al. A decellularized matrix hydrogel derived from human dental pulp promotes dental pulp stem cell proliferation, migration, and induced multidirectional differentiation in vitro. J Endod 2020;46(10):1438-1447.e5

14 Song JS, Takimoto K, Jeon M, Vadakekalam J, Ruparel NB, Diogenes A. Decellularized human dental pulp as a scaffold for regenerative endodontics. J Dent Res 2017;96(06):640-646

15 Matoug-Elwerfelli M, Duggal MS, Nazzal H, Esteves F, Raïf E. A biocompatible decellularized pulp scaffold for regenerative endodontics. Int Endod J 2018;51(06):663-673

16 Zhang W, Vazquez B, Oreadi D, Yelick PC. Decellularized tooth bud scaffolds for tooth regeneration. J Dent Res 2017;96(05): 516-523 